\title{
BESPREK
}

[1497] Gosse Blom $\dagger \&$ Siebren Dyk met ondersteuning van Wiebe Evert Zoethout, Graet Hylper Wordebook. Woordenboek van het Hindeloopers. Rige Fryske dialektstúdzjes 13. Ljouwert (2019), Afûk / Fryske Akademy, 1020 pp. ISBN 978-94-93159-00-6.

\section{In tsjok wurdboek}

It Graet Hylper Wordebook fan Gosse Blom en Siebren Dyk, mei stipe fan Wiebe Evert Zoethout, is in tsjok neislachwurk wurden. It is in wurdboek yn boekfoarm fan 1020 siden. Der is gjin digitale ferzje.

De foargonger, it Hylper wurdboek fan Gosse Blom út 1981, is folle tinner (182 siden). Dat hat in ynliedend diel fan 6 siden. It Graet Hylper Wordebook hat in ynliedend diel fan mar leafst 51 siden, mei ûnder oare in In Memoriam foar Gosse Blom, dy't ûnder it wurk oan it wurdboek ferstoarn is. It wurdboek wie doe al fierhinne klear. Fierder is der in oersjoch fan de boarnen dy't brûkt binne en in stik oer it Hylpersk. Nei it diel mei de trefwurden steane twa handige yndeksen: Nederlânsk-Hylpersk en Frysk-Hylpersk; dat de brûker kin de trefwurden ek fine fia it Nederlânsk of it Frysk.

It eigentlike wurdboek hat 696 siden. It tal trefwurden wurdt net neamd. Dat is skande en normaal sprutsen wurdt dat by wurdboeken wol dien. Neffens myn berekkening binne it likernôch 18.000 lemma's. Dat is it dûbelde fan it âlde wurdboek, dêr't it tal lemma's ek net neamd wurdt. Dat hat neffens myn berekkening likernôch 9200 lemma's. Likegoed is 18.000 frij beskieden foar in wurdboek fan dizze omfang. Der stiet faak wol in protte ynformaasje yn de lemma's. Yn it âlde wurdboek stiet by it Hylpersk trefwurd oer it algemien allinnich de Fryske en de Nederlânske oersetting, mei út en troch útlis of in foarbyld. Yn it nije wurdboek wurde folle mear foarbylden en folle mear taljochting jûn. It âlde wurdboek is mear in hânsum helpmiddel wylst it Graet Hylper Wordebook mear in wittenskiplik neislachwurk is.

\section{Doelgroep}

It is net hielendal dúdlik wa't de doelgroep is fan it wurdboek. Dy wurdt net eksplisyt neamd, allinnich even oanstipt yn de Inleiding op side 15, ûnder de kop Een nieuw woordenboek: 'Het woordenboek was immers niet alleen bestemd voor de Hindeloopers zelf, maar zou ook de taalkundig geïnteresseerden van dienst moeten zijn.'

Us Wurk 69 (2020), s. 92-96; https://doi.org/10.21827/5d4811b458089 
It wurdboek liket dus (ek) bedoeld te wêzen foar net-linguisten, foar de gewoane brûker. Yn it ynliedende diel, Enige bijzonderheden van uitspraak en grammatica van het Hindeloopers, komme je yndied in inkelde útlis tsjin dy't foar linguisten net nedich is mar dy't foar in net-linguist nuttich wêze kin. Sa stiet op side 75: 'Voegwoorden worden traditioneel verdeeld in nevenschikkende en onderschikkende. Nevenschikkende voegwoorden verbinden elementen van gelijke waarde, bijvoorbeeld [...] ' Linguisten witte dat wol, dy hawwe dy útlis net nedich. Mar folle faker komme je termen en formulearringen tsjin dy't foar in net-linguist ûnbekend wêze kinne, lykas 'diftongering' en 'palatalisatie'.

De lemma's sels biede ek ynformaasje dy't nijsgjirrich wêze kin foar de linguist/ûndersiker, mar dy't minder tagonklik is foar de gewoane brûker, lykas de ofkoartingen fan de boarnen en de inisjalen fan de skriuwers dêrfan. Wa't net thús is yn dy nammen, moat nei it oersjoch op side 23 oant en mei side 27. It hie makliker west as dy boarnen en nammen útskreaun wienen yn de lemma's sels.

De symboalen en de labels, faak yn it Latyn, lykje ek mear rjochte te wêzen op fakminsken as op de gewoane brûker, bygelyks a. - ante (voor), nom. prop. nomen proprietatum (eigennaam) [dat soe trouwens nomen proprium wêze moatte], s.l. - sine loco (zonder plaatsaanduiding).

In pear labels lykje sawat itselde út te drukken. Dêr hienen de makkers better kieze kinnen foar ien fan de twa: fig. - figuurlijk en overdr. - overdrachtelijk; inz. inzonderheid en m.n. - met name.

En de symboalen dy't brûkt binne, sille allegearre ek net foar elke net-linguist yntuïtyf dúdlik wêze en opsocht wurde moatte, lykas o - eenmaal mondeling of schriftelijk overgeleverd. Oare symboalen binne dúdliker: $\uparrow$ - uitgestorven, $\rightarrow$ - zie (ook) bij (verwijzing naar ander lemma binnen het woordenboek).

De faak wat stive manier fan formulearjen makket it wurdboek ek wat minder tagonklik, bygelyks yn de Inleiding, op side 14: 'De korte behandeling moge echter voldoende zijn om tot de conclusie te leiden dat het Hindeloopers weliswaar [...].' De Nederlânske oersetting en útlis yn de lemma's hie soms ek nofliker formulearre wêze kinnen, bygelyks by senteraesje - It mut oergaen óm de -, het moet overgaan vanwege de financiële toestand (de geldmiddelen zijn ontoereikend). Nofliker om te lêzen hie west: 'het kan niet doorgaan vanwege het geld'.

\section{In Memoriam}

Yn it ynliedende diel stiet in In Memoriam mei de tekst fan in grêfrede by de begraffenis fan Gosse Blom. Dy tekst is net oanpast foar dit wurdboek. No stiet der ynformaasje yn oer Gosse Blom dy't nijsgjirrich is foar famylje en freonen fan de ferstoarne, mar dy't te persoanlik is foar in publikaasje lykas dit wurdboek: 'Gosse is yn novimber 1975 op 'e Akademy kaam, yn it ramt fan wat dan sein waard "in regeling". Der waard ús troch de sjef wol ferteld dat er net foar $100 \%$ sûn wie [...].' De detaillearre ynformaasje oer de manier fan wurkjen is ek wat pynlik: 'Jo koene fernimme dat it him yn it begjin net tafoel, hy bleau nei myn smaak earst ek noch wolris wat te folle yn it stramyn fan syn earder wurdboekje hingjen, wylst er no 
eins folle djipper dolle moast.' De tekst is ek ynhelle troch de aktualiteit: 'Dat wurdboek komt der dus, ek al is it net mear dit jier.' Dat wie de sitewaasje doe't Gosse begroeven waard, mar no is it wurdboek der, it leit foar ús.

\section{Trefwurden}

De lemma's hawwe neist it Hylper trefwurd, de Fryske oersetting en de Nederlânske oersetting, faak noch útlis yn in Nederlânsk, en soms ferwizingen nei oare trefwurden en nei boarnen. Dêrmei jout it wurdboek moai ûndersyksmateriaal foar linguisten.

Yn it ynliedende diel stiet dat der âlde boarnen brûkt binne, mar dat der ek wurden opnaam binne út it Hylpersk sa't it no sprutsen wurdt. Dat fernimme je oan de kar fan de trefwurden. Sa binne der dúdlik histoaryske trefwurden: ambachtsskoele, boelgoed, boddebankjen (bêdsbankje; bankje voor de bedstee, gebruikt als zitplaats), êrmhús. Je komme ek typysk Hylper wurden en geografyske nammen tsjin: Achtfuttensteager (Achtvoetsteeg), blaaudammede (Naam van een stof met een bep. patroon (Hindelooper bont)), Meenskarwyken (Naam van de sloot die loopt van de Yndyk naar de Aensterfaart), peilskaelgeboutjen (peilschaalgebouwtje). Oan de oare kant binne der moderne en sprektalige trefwurden opnaam lykas bestand mei de betsjutting fan gegevensbestân, dashboard, ynternot, kómpjûter, lullifikaesje. Oare moderne wurden steane der dan wer net yn, lykas webside of de betsjutting kompjûtermûs by it trefwurd mús. Dat is ek net frjemd. Sa'n 18.000 lemma's is net in hiel grut oantal foar in wurdboek. Je kinne dan net ferwachtsje dat alle frekwinte wurden opnaam binne.

\section{Opbou en ynhâld lemma's}

Wurdsoarten

Guon wurden kinne ta mear wurdsoarten hearre, offhinklik fan hoe't se brûkt wurde. Dy wurdsoarten wurde yn it wurdboek oanjûn. Faak hie it ek goed west om it lemma dan neffens dy wurdsoarten op te splitsen. Dat makket it dúdliker foar de brûker op hokker ferskate manieren at je in wurd brûke kinne. Dat is yn dit wurdboek net altiten dien. Sa kin it trefwurd by prep \& adv (ferhâldingswurd en bywurd) wêze, mar wurdt de bywurdlike betsjutting net apart behannele. Dy is allinnich werjûn yn in pear útdrukkingen lykas Goo by wêze (goed bij zijn). By it trefwurd bûnt steane de trije wurdsoarten adj., adv. \& s (eigenskipswurd, bywurd en haadwurd) ek byinoar. It hie better west om yn alle gefallen bûnt as haadwurd apart te behanneljen, it leafst as in apart lemma.

\section{Foarbylden}

Soms is in foarbyldsin al genôch om it ferskil yn gebrûksmooglikheden fan in wurd te yllustrearjen, lykas by ferrúnewearje. Dit hat ûnder oare de Nederlânske oersetting stukmaken mar ek it net-oergonklike stukgaan. Dy net-oergonklike betsjutting is bysûnder en in foarbyldsin kin sjen litte hoe't dit tiidwurd net-oergonklik brûkt wurdt. By in trefwurd as ferlosse mei de fêste kombinaasje ferlosse fan (verlossen 
van), freegje ik my as brûker ôf oft je ferlosse yn it Hylpersk ek figuerlik brûke kinne, yn de sin fan ferlosse fan in ferfelende taak, fan dy ferfelende fint. As dat sa is, dan sjoch ik dat graach yn in foarbyldsin.

\section{Utlis}

De Hylper trefwurden binne oerset yn it Frysk en it Nederlânsk. Utdrukkingen hawwe allinnich in Nederlânske oersetting. Faak is der ek noch in útlis fan sa'n Nederlânsk wurd of Nederlânske útdrukking. Sa'n útlis wurdt ek jûn by Nederlânske wurden en útdrukkingen dy't algemien bekend binne. Yn dy gefallen is de útlis faak yngewikkelder as de oersetting en foeget dus neat ta. Sa fine we by it trefwurd wêze it foarbyld 'Jó ys daif'. De Nederlânske oersetting is dêr: 'zij is doof, zij heeft een stoornis van de gehoorfunctie'. It diel nei de komma is net nedich en yngewikkelder as it earste diel dêr't it in taljochting op wêze moat. Soks jildt ek foar de Nederlânske oersetting fan it foarbyld 'Waa syn boek ys dot?' yn itselde lemma wêze. De oersetting is hjir: 'wiens boek is dat?, aan wie behoort dat toe?'.

Der binne ek genôch gefallen dêr't de útlis wol funksjoneel is en faak tige nijsgjirrich. Sa fine we by matres: 'matres, eertijds houdster van een bewaar- of kleuterschool; ook wel gebruikt als aanduiding voor een onderwijzeres op een lagere school', of by seksjefergaedering, dêr't gjin oersetting by jûn wurdt, allinnich de Nederlânske útlis: 'Vergadering waaraan de raadsleden (minus de leden van het college van burgemeester en wethouders) en ambtenaren van de voormalige gemeente Hindeloopen deelnamen om de concept-begroting door te nemen.'

\section{Ferwizingen}

Yn de lemma's steane faak ferwizingen nei oare trefwurden, foaral synonimen. Dat kin nijsgjirrige ferbiningen opleverje mar soms binne it wol in hiele protte. Bygelyks yn it lemma neisên, allinnich al by de útdrukking Een neisên, mei de oersetting 'iem. op zijn falie geven, hem danig mishandelen', steane ferwizingen nei bêlch, blaet, bliksem, bröien, dónder, laezer, myter, ôfdrûgje, ôfraanselje, oftiggelje, offuge, raansel, raanselje, soademyter. De gewoane brûker sil dy net gau opsykje. Mar foar in ûndersiker kin it prachtich materiaal opleverje.

\section{Konklúzje}

It is in wurdboek mei in soad nijsgjirrige ynformaasje foar ûndersikers, mar foar de gewoane brûker is it net botte tagonklik. Yn de praktyk fan de moderne leksikografy wurdt in protte omtinken jûn oan brûkersfreonlikens. Ynliedingen wurde beheind en oersichtlik holden, oanwizingen foar it gebrûk steane yn oantreklike skema's mei pylkjes en kleurkes. Lemma's hawwe in yndieling dy't noflik lêst en wurde noch oantrekliker makke mei kleuren. Labels binne sa begryplik mooglik, leafst net yn it Latyn en foarbylden en útlis wurde yn tagonklike taal formulearre. Der wurdt oer it algemien ek in digitale ferzje fan in wurdboek makke, te brûken op de tablet of tillefoan of, bygelyks foar oersetters, op de kompjûter. Dy prinsipes fan de moderne leksikografy binne yn dit wurdboek net tapast. In ûndersiker is nei 
alle gedachten bliid mei de wiidweidige ynlieding en de wiidweidige ynformaasje yn de lemma's, mar foar de gewoane brûker is it allegearre frijwat dreech en ûnoantreklik. Boppedat sil de brûker in wurd faak net fine. It is gjin wurdboek mei de meast frekwinte wurden, lykas wenstich is yn wurdboeken mei in beheind tal trefwurden. De kar fan de lemma's liket bepaald te wêzen troch wat der beskikber wie út de âlde boarnen oan de iene kant, en oan de oare kant troch wat de makker oppikt hat fan it hjoeddeistige Hylpersk. It is in wat ûnlykwichtich ferskaat fan trefwurden. De priis, $€ 65$, nûget foar in gewoane brûker ek net út om dit wurdboek te keapjen. Dat is skande, want it is moai foar it Hylpersk dat sa'n wurdboek der is.

Martha Hofman

mahofman@hetnet.nl 